\title{
On optimal dividend strategies with a random time horizon *
}

\author{
Hansjörg Albrecher $^{\dagger, \ddagger}$ And Stefan Thonhauser ${ }^{\dagger}$ \\ ${ }^{\dagger}$ Department of Actuarial Science, Faculty of Business and Economics, \\ University of Lausanne, \\ Quartier UNIL-Dorigny, Bâtiment Extranef, 1015 Lausanne, Switzerland. \\ ¥ Swiss Finance Institute, University of Lausanne
}

\begin{abstract}
For the classical compound Poisson surplus process of an insurance portfolio we investigate the problem of how to optimally pay out dividends to shareholders if the criterion is to maximize the expected discounted dividend payments until the time of ruin or a random time horizon, whichever is smaller. We explicitly solve this problem for an exponential time horizon and exponential claim sizes. Furthermore, we study the case of an Erlang(2) time horizon by introducing an external state process and derive the solution under the assumption that the external state process is observable. The results are illustrated by numerical examples.
\end{abstract}

\section{Introduction and Model}

Let $(\Omega, \mathcal{F}, \mathbb{P})$ be a probability space, sufficiently large to carry all the stochastic quantities that will be introduced in the sequel. Consider the classical collective risk model (also called Cramér-Lundberg model) for the surplus process $R=\left(R_{t}\right)_{t \geq 0}$ of an insurance portfolio, i.e.

$$
R_{t}=x+c t-\sum_{i=1}^{N_{t}} Y_{i},
$$

with initial surplus $R_{t}=x \geq 0$, a homogeneous Poisson process $N=\left(N_{t}\right)_{t \geq 0}$ with parameter $\lambda>0$ and a sequence of independent identically distributed claim amounts $\left\{Y_{i}\right\}_{i \in \mathbb{N}}$ with $F_{Y}(x)=\mathbb{P}\left(Y_{1} \leq x\right)$, where $\left\{Y_{i}\right\}_{i \in \mathbb{N}}$ and $N=\left(N_{t}\right)_{t \geq 0}$ are independent. Here $c>\lambda \mathbb{E}(Y)$ is a constant premium intensity.

Let $\tau=\inf \left\{t>0 \mid R_{t}<0\right\}$ denote the time of ruin. One of the classical concepts in risk theory is the probability of ruin $\psi(x)=\mathbb{P}_{x}(\tau<\infty)$, which is a measure for the safety of the insurance portfolio, see Rolski et al. [16] and Asmussen \& Albrecher [4] for an overview of ruin probabilities and related quantities.

In [10], de Finetti proposed the use of expected discounted dividend payments until ruin as a different criterion for measuring the performance of an insurance portfolio. This leads to a stochastic control problem of how to optimally pay out dividends in order to maximize this quantity. In the present model, an admissible dividend strategy is a càglàd non-decrasing process $L=\left(L_{t}\right)_{t \geq 0}$, and the controlled reserve becomes

$$
R_{t}^{L}=x+c t-\sum_{i=1}^{N_{t}} Y_{i}-L_{t} .
$$

\footnotetext{
${ }^{*}$ Supported by the Swiss National Science Foundation Project 200021-124635/1.
} 
The process $L$ describes the cumulated dividend payments over time. One has the restrictions $\Delta L_{t} \leq R_{t-}^{L}$ (i.e. dividend payments can not lead to ruin) and $L_{t}=L_{\tau^{L}}$ for $t \geq \tau^{L}$ (i.e. no payments after the event of ruin). Here $\tau^{L}$ denotes the time of ruin of $R^{L}$. The value of an admissible dividend strategy $L$ is given by

$$
\widetilde{V}(x, L)=\mathbb{E}_{x}\left(\int_{0}^{\tau^{L}} e^{-\delta t} d L_{t}\right),
$$

where $\delta>0$ is a constant discount rate (or simply a rate that reflects the preference to receive payments earlier rather than later).

The stochastic control problem to determine the optimal dividend strategy has been studied under various model assumptions and constraints, see Albrecher \& Thonhauser [3] and Avanzi

[6] for recent surveys. One should note that that there are similarities, but also subtle differences to other stochastic control problems in mathematical finance.

In this contribution, we would like to address a variation of the classical dividend problem. Usually the time horizon of the dividend maximization problems is the time of ruin (unless one includes the possibility of reinvestments whenever the surplus turns negative, in which case the time horizon is infinite, see Kulenko \& Schmidli [14]). An exception is the paper of Grandits [13], who deals with the problem on a finite time horizon for a diffusion approximation of the risk reserve process $R_{t}$ (i.e. dividends can be collected up to the minimum of the time of ruin and a prespecified fixed time horizon). Such a finite time horizon is a reasonable assumption in view of finite planning horizons for portfolio management. However, the introduction of the finite time horizon makes the problem technically highly involved, and one can expect this to be even worse when generalizing the underlying portfolio process to a compound Poisson process with drift (which is the more natural model for the insurance context). Here we propose a different route towards the solution of the dividend problem in the classical collective risk model with finite horizon, namely to randomize the deterministic time horizon. Such a randomization was successfully used in other finite horizon problems in insurance and mathematical finance (see for instance Asmussen et al. [5] and Carr [8]) and the results served as a reasonable approximation to the original problem. In Section 2 we will focus on the case of an exponential time horizon. In Section 3 we will then study the extension to an Erlang(2) time horizon, which is a crucial step towards an approximation for the solution of the optimal dividend problem in the Cramér-Lundberg model with deterministic finite time horizon. In Section 4 we finally indicate some further research directions.

\section{Exponential time horizon}

Let us assume in this section that the time horizon is an exponentially distributed random variable $\zeta \sim \operatorname{Exp}(\gamma)$, independent of $\left\{Y_{i}\right\}$ and $N$. In other words, we apply exponential killing at a constant rate $\gamma$ to the risk reserve and want to study its effect on the dividend maximization problem. Under this assumption the value of a strategy $L$ is given by

$$
\widetilde{V}(x, L)=\mathbb{E}_{x}\left(\int_{0}^{\tau^{L} \wedge \zeta} e^{-\delta t} d L_{t}\right) .
$$

In view of the setting of the original de Finetti problem, where the emphasis is on maximizing profit, it is reasonable to assume that if the process is stopped at a time $\zeta<\tau^{L}$, then the remaining positive reserve can be paid out as dividends as well (otherwise the problem is 
simpler, see Remark 2.1). This leads to the modification

$$
V(x, L)=\mathbb{E}_{x}\left(\int_{0}^{\tau^{L} \wedge \zeta} e^{-\delta t} d L_{t}+e^{-\delta \zeta} R_{\zeta}^{L} I_{\left\{\zeta<\tau^{L}\right\}}\right) .
$$

Let $\mathcal{L}$ be the set of admissible dividend strategies with the constraint that there are no payments after time $\zeta \wedge \tau^{L}$ and that the reserve process is stopped from $\zeta \wedge \tau^{L}$ onwards. The value function of the dividend maximization problem under the random time horizon is then given by

$$
V(x)=\sup _{L \in \mathcal{L}} V(x, L)
$$

We first observe that, due to $P(\zeta>t)=e^{-\gamma t}$, for an admissible strategy $L$ in the infinite horizon problem the strategy $\widetilde{L}=\left(e^{-\gamma t} L_{t}\right)_{t \geq 0}$ corresponds to an admissible strategy for the killed reserve. Secondly we have that $P(\zeta \in d t)$ is $\gamma e^{-\gamma t} d t$, so that the expectation of $e^{-\delta \zeta} R_{\zeta}^{L} I_{\left\{\zeta<\tau^{L}\right\}}$ with respect to $\zeta$ translates to $\int_{0}^{\tau^{L}} \gamma e^{-(\delta+\gamma) t} R_{t}^{L} d t$. As a consequence we can rewrite $V(x, L)$ in the form

$$
V(x, L)=\mathbb{E}_{x}\left(\int_{0}^{\tau^{L}} e^{-(\delta+\gamma) t} d L_{t}\right)+\mathbb{E}_{x}\left(\int_{0}^{\tau^{L}} e^{-(\delta+\gamma) t} \gamma R_{t}^{L} d t\right) .
$$

Similar arguments for interchanging killing and discounting apply for state-dependent killing rates, see for instance Davis [9, Theorem 31.9]. We can now write down (for the moment informally) the Hamilton-Jacobi-Bellman (HJB) equation associated to $V$ in terms of a suitable function $f:[0, \infty) \rightarrow[0, \infty)$ :

$$
\max \left\{c f^{\prime}(x)+\lambda \int_{0}^{x} f(x-y) d F_{Y}(y)-(\delta+\gamma+\lambda) f(x)+\gamma x, 1-f^{\prime}(x)\right\}=0 .
$$

Note that $V(x)=0$ for $x<0$. The derivation of (1) follows standard arguments, see Fleming \& Soner [11] or Schmidli [17].

If we define $\Delta$ as the death state, the generator of the killed risk reserve (as a part of (1)) is given by

$$
c f^{\prime}(x)+\lambda \int_{0}^{x} f(x-y) d F_{Y}(y)-(\gamma+\lambda) f(x)+\gamma f(\Delta) .
$$

In our situation the value function evaluated at the death state is just equal to the terminal payment of the reserve.

Remark 2.1. Considering the killing procedure for the dividend problem without possible terminal payment (i.e. using $\widetilde{V}(x, L)$ instead of $V(x, L))$ leads to the original problem without killing, but with the modified discounting factor $\delta+\gamma$ instead of $\delta$. So in this case the effect of a finite random time horizon is just a simple shift of the discounting parameter (the solution to this problem can correspondingly be found in Azcue \& Muler [7] and Schmidli [17]).

\subsection{Explicit solution in case of exponential claims}

In order to quantitatively study the consequences of the finite (random) time horizon on the dividend problem, we will restrict ourselves in the following to the case of exponentially distributed claim amounts $\left(Y_{1} \sim \operatorname{Exp}(\alpha)\right)$. Under this assumption one can expect an explicit 
expression for the value function and the optimal strategy. Concretely, we can then construct a concave solution to (1) with the consequence that a so-called barrier strategy is optimal (at the end of the section we will give a verification result for a formal justification). A barrier strategy with barrier $b \geq 0$ pays every excess of the reserve over $b$ immediately as dividend.

Barrier in zero. Suppose a barrier strategy with level $b=0$ is applied and denote the associated dividend process by $L^{0}$, then we have

$$
V\left(x, L^{0}\right)=x+\int_{0}^{\infty} \int_{0}^{T} e^{-(\delta+\gamma) t} c d t \lambda e^{-\lambda T} d T=x+\frac{c}{\delta+\gamma+\lambda} .
$$

Define the constant $A=(\gamma+\delta+\lambda)-\alpha c$.

Lemma 2.1. For $Y_{1} \sim \operatorname{Exp}(\alpha), V\left(x, L^{0}\right)$ is a solution to (1) either if $A \geq 0$ or $A<0$ and $\alpha \lambda c-(\delta+\lambda)(\gamma+\delta+\lambda) \leq 0$.

Proof. We only need to check if

$$
c V^{\prime}\left(x, L^{0}\right)+\lambda \int_{0}^{x} V\left(x-y, L^{0}\right) d F_{Y}(y)-(\delta+\gamma+\lambda) V\left(x, L^{0}\right)+\gamma x \leq 0
$$

for $x \geq 0$. Define $G(x):=\lambda\left(e^{-\alpha x}-1\right) A-x \alpha \delta(\gamma+\delta+\lambda)$, then the left hand side of (2) can be written as

$$
\frac{G(x)}{\alpha(\delta+\gamma+\lambda)}
$$

so that we only need to check the sign of $G(x)$.

If $A>0$, then $G(x)$ is decreasing, with $G(0)=0$ as its maximum.

If $A<0$, then $G(x)$ is strictly concave. Furthermore $G^{\prime}(0) \leq 0$ which is equivalent to $\alpha \lambda c-(\delta+\lambda)(\gamma+\delta+\lambda) \leq 0$. Hence $G(0)=0$ is a maximum of $G$ for $x \geq 0$.

Barrier larger than zero. From now on assume $A<0$ and $\alpha \lambda c-(\delta+\lambda)(\gamma+\delta+\lambda)>0$. Trying the construction of a concave solution $f:[0, \infty) \rightarrow[0, \infty)$ to (1) implies the existence of a point $x^{*}$ such that $f^{\prime}(x) \geq 1$ for $0 \leq x \leq x^{*}$ and $f^{\prime}(x)<1$ for $x^{*}<x$.

At first we are going to construct a differentiable solution $f_{b}$ with $b>0$ to the problem:

$$
\begin{cases}c f_{b}^{\prime}(x)+\lambda \int_{0}^{x} f_{b}(x-y) d F_{Y}(y)-(\delta+\gamma+\lambda) f_{b}(x)+\gamma x=0, & 0 \leq x \leq b, \\ x-b+f_{b}(b), & x>b .\end{cases}
$$

Correspondingly, $f_{b}(x)=V\left(x, L^{b}\right)$ refers to the expected discounted dividends until ruin under a barrier strategy with barrier $b$. Some calculations show that for $0 \leq x \leq b$ the function

$$
f_{b}(x)=A_{1}(b) e^{R_{1} x}+A_{2}(b) e^{R_{2} x}+\frac{\gamma x}{\gamma+\delta}+\frac{\gamma(\alpha c-\lambda)}{\alpha(\gamma+\delta)^{2}},
$$

together with the linear continuation in $b$ is a differentiable solution to (3). The corresponding coefficients are given by

$$
\begin{aligned}
& A_{1}(b)=\frac{\left(R_{1}+\alpha\right)\left(\alpha^{2} \delta(\gamma+\delta)-R_{2}\left(R_{2}+\alpha\right) \gamma A e^{R_{2} b}\right)}{\alpha^{2}(\gamma+\delta)^{2}\left(R_{1}\left(R_{1}+\alpha\right) e^{R_{1} b}-R_{2}\left(R_{2}+\alpha\right) e^{R_{2} b}\right)} \\
& A_{2}(b)=-\frac{\left(R_{2}+\alpha\right)\left(\alpha^{2} \delta(\gamma+\delta)-R_{1}\left(R_{1}+\alpha\right) \gamma A e^{R_{1} b}\right)}{\alpha^{2}(\gamma+\delta)^{2}\left(R_{1}\left(R_{1}+\alpha\right) e^{R_{1} b}-R_{2}\left(R_{2}+\alpha\right) e^{R_{2} b}\right)}
\end{aligned}
$$


and the exponents are

$$
R_{1}=\frac{A-\sqrt{A^{2}+4 \alpha c(\gamma+\delta)}}{2 c}<0, \quad R_{2}=\frac{A+\sqrt{A^{2}+4 \alpha c(\gamma+\delta)}}{2 c}>0 .
$$

The principle of smooth fit suggests to derive the maximizing level $b^{*}$ by the condition $f_{b^{*}}^{\prime \prime}\left(b^{*}\right)=$ 0 . Intuitively this is in accordance with pasting the linear function with the highest curve from below. Define $H(b):=f_{b}^{\prime \prime}(b)$.

Lemma 2.2. If $A<0$ and $\alpha \lambda c-(\delta+\lambda)(\gamma+\delta+\lambda)>0$, then there exists a unique positive solution $b^{*}$ to $H\left(b^{*}\right)=0$.

Proof. By our assumption, $H(0)=-\frac{\alpha \lambda c-(\delta+\lambda)(\gamma+\delta+\lambda)}{c(\delta+\gamma+\lambda)}<0$. Furthermore, $\lim _{b \rightarrow \infty} H(b)=$ $\frac{\delta R_{2}}{\delta+\gamma}>0$. Since $R_{1}+\alpha>0$, there is no positive root of the denominator and therefore $H(b)$ is continuous for $b \geq 0$. One can further show by straight-forward but cumbersome calculations that $H^{\prime}(b)>0$, which proves that there exists a unique $b^{*}>0$ such that $H\left(b^{*}\right)=0$.

Remark 2.2. One observes that at $b=b^{*}$ the coefficients simplify as follows:

$$
A_{1}\left(b^{*}\right)=\frac{\delta R_{2} e^{-b^{*} R_{1}}}{R_{1}\left(R_{2}-R_{1}\right)(\delta+\gamma)}, \quad A_{2}\left(b^{*}\right)=\frac{\delta R_{1} e^{-b^{*} R_{2}}}{R_{2}\left(R_{1}-R_{2}\right)(\delta+\gamma)} .
$$

In particular, we have

$$
f_{b^{*}}\left(b^{*}\right)=\frac{\alpha(c+\gamma b)-(\delta+\lambda)}{\alpha(\delta+\gamma)}
$$

Lemma 2.3. For $Y_{1} \sim \operatorname{Exp}(\alpha)$ and under the conditions $A<0$ and $\alpha \lambda c-(\delta+\lambda)(\gamma+\delta+\lambda)>0$, the function $f_{b^{*}}(x)$ defined by (4) and (3) with $b^{*}$ from Lemma 2.2 is an increasing concave solution to (2).

Proof. Since $A<0$ we have that $A_{1}\left(b^{*}\right)<0$ and from the definition of $b^{*}$,

$$
R_{1}^{2} A_{1}\left(b^{*}\right) e^{R_{1} b^{*}}=-R_{2}^{2} A_{2}\left(b^{*}\right) e^{R_{2} b^{*}},
$$

which implies $A_{2}\left(b^{*}\right)>0$, such that in total for $0 \leq x \leq b^{*}$ we have $f_{b^{*}}^{\prime}(x)>0$. Furthermore this implies that $f_{b^{*}}^{\prime \prime \prime}(x)>0$ for $0 \leq x \leq b^{*}$, since $f_{b^{*}}^{\prime \prime}\left(b^{*}\right)=0$ the function is concave, and that $f_{b^{*}}^{\prime}\left(b^{*}\right)=1$ is a strict minimum of the first derivative. Because of the linear continuation above $b^{*}$ these properties remain valid for $x>b^{*}$ as well.

By construction, the function $f_{b^{*}}$ solves $(2)$ on $\left(0, b^{*}\right]$. Since on $\left(b^{*}, \infty\right)$ we have $f_{b^{*}}^{\prime}(x)=1$ we finally need to check if

$$
\begin{aligned}
c+\lambda \int_{0}^{x-b^{*}}\left(x-b^{*}+f_{b^{*}}\left(b^{*}\right)-y\right) \alpha e^{-\alpha y} d y-(\delta & +\gamma+\lambda)\left(x-b^{*}+f_{b^{*}}\left(b^{*}\right)\right) \\
& +\gamma x+\lambda \int_{x-b^{*}}^{x} f_{b^{*}}(x-y) \alpha e^{-\alpha y} d y \leq 0 .
\end{aligned}
$$

From $\lambda \int_{x-b^{*}}^{x} f_{b^{*}}(x-y) \alpha e^{-\alpha y} d y \leq \lambda f_{b^{*}}\left(b^{*}\right)\left(e^{-\alpha\left(x-b^{*}\right)}-e^{-\alpha x}\right),(5)$ and using $\alpha \lambda c-(\delta+\lambda)(\gamma+$ $\delta+\lambda)>0$, we obtain the result.

The following standard verification theorem gives the formal basis for the above calculations. Since in both parameter constellations above, we have concave and increasing solutions to (2) which correspond to admissible dividend strategies, we are going to prove: 
Theorem 2.4. Let $h: \mathbb{R} \rightarrow \mathbb{R}^{+}$with $h(x)=0$ for $x<0$ be a differentiable, concave and increasing solution to (2) which corresponds to an admissible dividend strategy $L^{*}=\left(L_{t}^{*}\right)_{t \geq 0}$. Then $h=V$, and the strategy $L^{*}$ is optimal.

Proof. Let $L=\left(L_{t}\right)_{t \geq 0}$ be an arbitrary admissible dividend strategy ( $L^{c}$ denoting its absolutely continuous component). Then for $t>0$ we can write

$$
\begin{aligned}
& e^{-(\delta+\gamma) t \wedge \tau^{L}} h\left(R_{t \wedge \tau^{L}}^{L}\right)= \\
& h(x)+\int_{0}^{t \wedge \tau^{L}} e^{-(\delta+\gamma) s}\left[c h^{\prime}\left(R_{s-}^{L}\right)+\lambda \int_{0}^{R_{s-}^{L}} h\left(R_{s-}^{L}-y\right) \alpha e^{-\alpha y} d y-(\delta+\gamma+\lambda) h\left(R_{s-}^{L}\right)\right] d s \\
& \quad-\int_{0}^{t \wedge \tau^{L}} e^{-(\delta+\gamma) s} h^{\prime}\left(R_{s-}^{L}\right) d L_{s}^{c}-\sum_{0 \leq s \leq t \wedge \tau^{L} \wedge R_{s+}^{L} \neq R_{s}^{L}} e^{-(\delta+\gamma) s}\left[h\left(R_{s+}^{L}\right)-h\left(R_{s}^{L}\right)\right] .
\end{aligned}
$$

Since $R_{s+}^{L}-R_{s}^{L}=L_{s}-L_{s+}$ and $1-h^{\prime}(x) \geq 0$ we have

$$
\begin{aligned}
& -\int_{0}^{t \wedge \tau^{L}} e^{-(\delta+\gamma) s} h^{\prime}\left(R_{s-}^{L}\right) d L_{s}^{c}-\sum_{0 \leq s \leq t \wedge \tau^{L} \wedge R_{s+}^{L} \neq R_{s}^{L}} e^{-(\delta+\gamma) s}\left[h\left(R_{s+}^{L}\right)-h\left(R_{s}^{L}\right)\right] \leq \\
& -\int_{0}^{t \wedge \tau^{L}} e^{-(\delta+\gamma) s} d L_{s}^{c}-\sum_{0 \leq s \leq t \wedge \tau^{L} \wedge R_{s+}^{L} \neq R_{s}^{L}} e^{-(\delta+\gamma) s}\left[L_{s+}-L_{s}\right]=-\int_{0}^{t \wedge \tau^{L}} e^{-(\delta+\gamma) s} d L_{s} .
\end{aligned}
$$

Together with

$$
c h^{\prime}\left(R_{s-}^{L}\right)+\lambda \int_{0}^{R_{s-}^{L}} h\left(R_{s-}^{L}-y\right) \alpha e^{-\alpha y} d y-(\delta+\gamma+\lambda) h\left(R_{s-}^{L}\right) \leq-\gamma R_{s-}^{L},
$$

we arrive at

$$
\begin{aligned}
\mathbb{E}_{x}\left(e^{-(\delta+\gamma) t \wedge \tau^{L}} h\left(R_{t \wedge \tau^{L}}^{L}\right)\right) & \\
& +\mathbb{E}_{x}\left(\int_{0}^{t \wedge \tau^{L}} e^{-(\delta+\gamma) s} d L_{s}+\int_{0}^{t \wedge \tau^{L}} e^{-(\delta+\gamma) s} \gamma R_{s-}^{L} d s\right) \leq h(x),
\end{aligned}
$$

where we can take $R_{s}^{L}$ instead of $R_{s-}^{L}$ in the last integral, since with probability zero there occurs a claim exactly at time $\zeta$.

Now we use dominated convergence for the limit $t \rightarrow \infty$ to deduce:

$$
V(x, L) \leq h(x) .
$$

Using strategy $L^{*}$ we derive an equality in (6) which proves its optimality.

We summarize our results in the following theorem.

Theorem 2.5. If $A \geq 0$ or $A<0$ and $\alpha \lambda c-(\delta+\lambda)(\gamma+\delta+\lambda) \leq 0$, then the value function is given by

$$
V(x)=x+\frac{c}{\delta+\gamma+\lambda} .
$$

The optimal dividend strategy is then a barrier strategy with barrier $b^{*}=0$.

If on the other hand $A<0$ and $\alpha \lambda c-(\delta+\lambda)(\gamma+\delta+\lambda)>0$, then the value function is $V(x)=f_{b^{*}}(x)$ where $f_{b^{*}}$ is determined by (3) and (4). In this case the optimal dividend strategy is a barrier strategy with barrier $b^{*}>0$, which is determined in Lemma 2.2. 


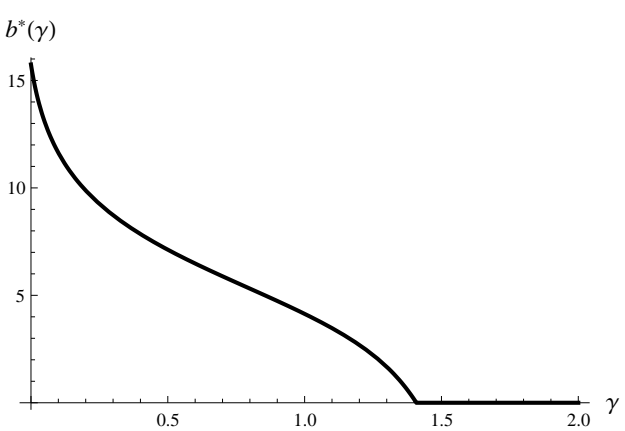

Figure 1: $b^{*}$ as function of $\gamma$

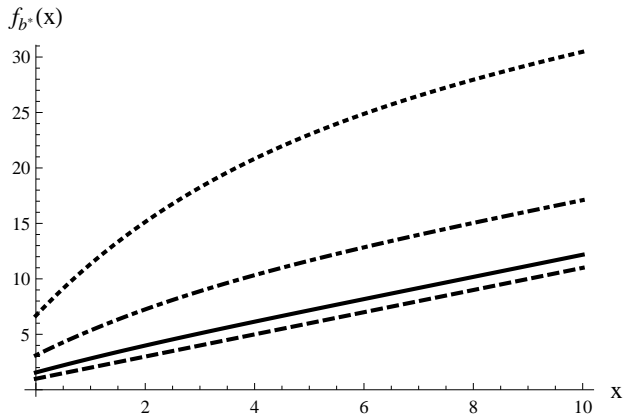

Figure 2: $f_{b^{*}}$ for $\gamma=0,0.1,0.5,1.5$

Example. Figure 1 shows the optimal barrier as a function of $\gamma$ for the parameter set $c=$ $\overline{6.5, \alpha=1}, \lambda=5$ and $\delta=0.04$. Due to the conditions of Theorem 2.5 it tends to zero if $\gamma$ increases. Figure 2 displays the value function for different values of $\gamma$. The dotted line corresponds to $\gamma=0$ with $b^{*}(0)=15.7448$, the dotted-dashed line to $\gamma=0.1$ with $b^{*}(0.1)=11.6348$, the solid line to $\gamma=0.5$ with $b^{*}(0.5)=7.1230$ and finally the dashed line to $\gamma=1.5$ with $b^{*}(1.5)=0$. As expected the value function decreases with increasing $\gamma$ (due to expected earlier killing of the surplus process).

Remark 2.3. If the underlying process $R_{t}$ is a diffusion, the above result will again hold, with analogous barrier strategies to be optimal. This can either be shown directly by similar calculations, or also by a suitable limiting procedure of the compound Poisson process (see e.g. Albrecher, Bäuerle \& Thonhauser [1] for a related procedure in another control problem).

Remark 2.4. The HJB equation corresponding to $\widetilde{V}(x)=\sup _{L \in \mathcal{L}} \widetilde{V}(x, L)$ is given by

$$
\max \left\{c f^{\prime}(x)+\lambda \int_{0}^{x} f(x-y) d F_{Y}(y)-(\delta+\gamma+\lambda) f(x), 1-f^{\prime}(x)\right\}=0 .
$$

Although in the infinite horizon case $\gamma=0$, barrier strategies are not always optimal beyond exponential claims (see Loeffen \& Renaud [15] for more detailed criteria) it is surprisingly hard to identify explicit optimal non-barrier strategies. There is one concrete example in Azcue \& Muler [7] for Erlang(2) claims, in which a barrier strategy is not optimal: For the parameters $F_{Y}(y)=1-(1+y) e^{-y}, \lambda=10, \delta=0.1, c=21.4$ the value function turns out to be

$$
\widetilde{V}(x)=\left\{\begin{array}{cc}
x+2.11881, & 0 \leq x \leq 1.80303 \\
g(x), & 1.80303<x \leq 10.2162, \\
x+2.45582, & 10.2162<x
\end{array}\right.
$$

with

$$
g(x)=11.2571 e^{0.039567 x}-9.43151 e^{-0.079355 x}+0.094314 e^{-1.48825 x} .
$$

The optimal strategy is of band type, i.e. for $0 \leq x \leq 1.80303$ a barrier strategy in zero is used, for $1.80303<x \leq 10.2162$ no dividends are paid and for $10.2162<x$ a barrier strategy with height 10.2162 is applied.

Since band strategies are sensitive to slight modifications of the model (see e.g. Albrecher et al. [1]), let us check the effect of $\gamma>0$ on the optimal strategy with respect to the infinite horizon case $\gamma=0$. Figures 3 and 4 illustrate that for the same parameter set, but $\gamma=0.1$, a 


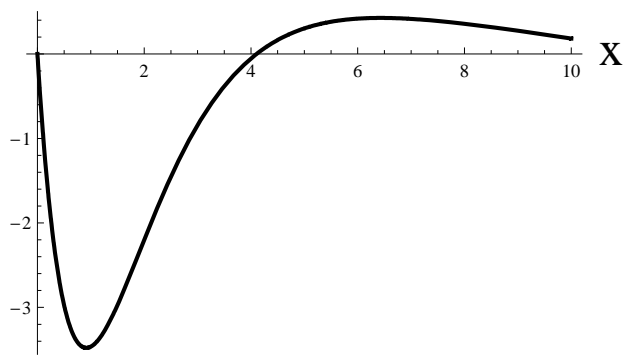

Figure 3: IDE part of HJB eq. for $f_{0}$ and $\gamma=0$

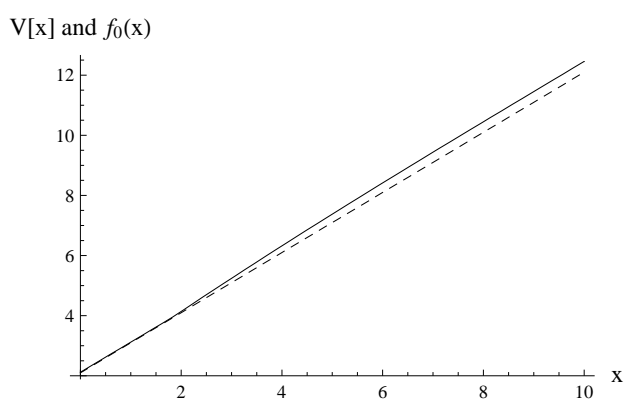

Figure 5: $\widetilde{V}(x)$ for $\gamma=0, f_{0}(x)$ for $\gamma=0.1$

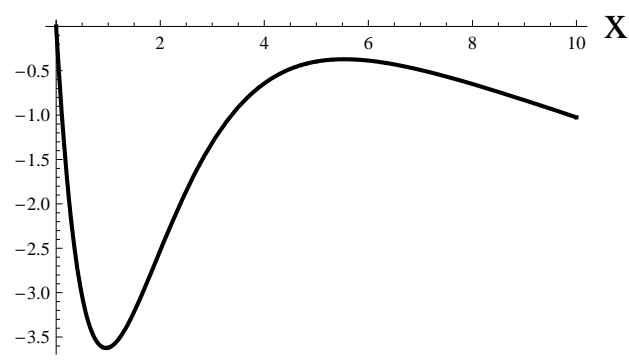

Figure 4: IDE part of HJB eq. for $f_{0}$ and $\gamma=0.1$

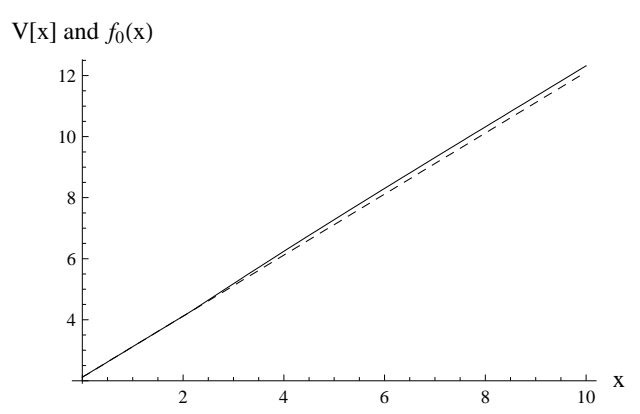

Figure 6: $\widetilde{V}(x)$ and $f_{0}(x)$ for $\gamma=0.01$

barrier strategy in zero is already optimal (i.e., the bands collapse to a barrier in zero). Figure 3 shows the left part of the maximum in the HJB equation ("IDE part") evaluated at the function $x+\frac{c}{\delta+\lambda}$ (which corresponds to a barrier in zero) for $\gamma=0$, which clearly shows that it can not be the value function. On the other hand, Figure 4 shows the IDE part evaluated at the value of a barrier strategy equal to zero for $\gamma=0.1$, which shows that now $x+\frac{c}{\delta+\lambda+\gamma}$ solves the HJB equation. Figure 5 shows $\widetilde{V}(x)$ in the case $\gamma=0$ (solid line) and $f_{0}=x+\frac{c}{\delta+\gamma+\lambda}$ which is the value function for $\gamma=0.1$ (dashed line).

On the other hand, for $\gamma=0.01$ a band strategy is still optimal (namely to use a barrier in zero for $x \in[0,2.18793]$, pay no dividends for $x \in(2.18793,9.07912]$ and use another barrier in 9.07912 for $x>9.07912$ ). Figure 6 depicts the associated value function (solid line) together with the performance under the barrier strategy $f_{0}$ (dashed line).

\section{Erlang time horizon}

A natural extension of an exponential random variable for the random time horizon is an $\operatorname{Erlang}(n, \gamma)$ distributed random variable. Although one then loses the attractive lack-ofmemory property of $\zeta$, choosing $n>1$ is a substantial step forward towards the case of deterministic finite time horizon, since for fixed expected value the Erlang $(n)$ distribution converges weakly to a point mass at $\mathbb{E}(\zeta)$. This so-called Erlangization method was for instance exploited to approximate finite-horizon ruin probabilities in Asmussen et al. [5], where it turned out that small values of $n$ often already give very good approximations for the deterministic fixed time horizon counterpart. As mentioned in the introduction, the dividend maximization problem with a finite time horizon was up to now only studied for a diffusion 
risk reserve process in Grandits [13]. It turns out that even for this relatively simple case (with constant coefficients), the problem becomes technically very complicated and it is only possible to construct $\varepsilon$-optimal strategies, given by time-dependent barrier strategies.

In this section we will study the case $\zeta \sim \operatorname{Erlang}(2, \gamma)$ to see the effect of the Erlangization on the calculations and to provide an alternative route towards finite horizon dividend problems in the framework of the classical risk model. In principle, the methodology will be the same when using larger values for $n$, but for reasons of compactness we restrict ourselves to the case $n=2$. As in the previous section, we consider exponentially distributed claim amounts. In the sequel, the flow of information over time is supposed to be given by the filtration generated by the reserve process and an external state process.

\subsection{State-dependent barrier strategies}

Let us recall the simple property that a random variable $\zeta \sim \operatorname{Erlang}(2, \gamma)$ can be written as $\zeta \sim \zeta_{1}+\zeta_{2}$ where $\zeta_{1}, \zeta_{2} \stackrel{i i d}{\sim} \operatorname{Exp}(\gamma)$. Suppose the process $\varepsilon=\left(\varepsilon_{t}\right)_{t \geq 0}$ is an external state process governed by the rule

$$
\varepsilon_{t}= \begin{cases}1, & 0 \leq t<\zeta_{1} \\ 2, & \zeta_{1} \leq t<\zeta_{1}+\zeta_{2} \\ \Delta, & t \geq \zeta_{1}+\zeta_{2}\end{cases}
$$

I.e., at time zero it starts in state 1 , after a time interval of length $\zeta_{1}$ it moves to state 2 and after a further time interval of length $\zeta_{2}$, it moves to the death state $\Delta$. Note that this is a particular form of a Markov switching problem, where the underlying process has the same dynamics across states, but the control at time $t$ depends on the value of $\varepsilon_{t}$. In particular, we assume here that we can observe the process $\left(\varepsilon_{t}\right)$, whereas in practice one will not be able to determine the current state of the process (this is somewhat akin to the approach of Albrecher \& Hartinger [2] in a different context). Recently, Sotomayor \& Cadenillas [18] considered a Markov switching environment for a diffusion process with state-dependent coefficients.

Note that the two-dimensional process $\left(R_{t}, \varepsilon_{t}\right)$ has the strong Markov property. Let $L=$ $\left(L_{t}\right)_{t \geq 0}$ be an admissible strategy, the value of which, when starting in state 1 , is defined as

$$
V_{1}(x, L)=\mathbb{E}_{(x, 1)}\left(\int_{0}^{\tau^{L} \wedge \zeta} e^{-\delta t} d L_{t}+e^{-\delta \zeta} R_{\zeta}^{L} I_{\left\{\zeta<\tau^{L}\right\}}\right) .
$$

If starting in state 2 we write,

$$
V_{2}(x, L)=\mathbb{E}_{(x, 2)}\left(\int_{0}^{\tau^{L} \wedge \zeta_{2}} e^{-\delta t} d L_{t}+e^{-\delta \zeta_{2}} R_{\zeta_{2}}^{L} I_{\left\{\zeta<\tau^{L}\right\}}\right) .
$$

When starting in state 2 , there is only one exponentially distributed time span left until moving to the death state, and so $V_{2}(x, L)$ coincides with the value of $L$ under exponential killing from Section 2.

The next lemma connects $V_{1}(x, L)$ with $V_{2}(x, L)$.

Lemma 3.1. Let $L=\left(L_{t}\right)_{t \geq 0}$ be an admissible dividend strategy such that $\left(R^{L}, \varepsilon\right)$ is a strong Markov process. Then

$$
V_{1}(x, L)=\mathbb{E}_{(x, 1)}\left(\int_{0}^{\tau^{L}} e^{-(\delta+\gamma) t} d L_{t}+\int_{0}^{\tau^{L}} e^{-(\delta+\gamma) t} \gamma V_{2}\left(R_{t}^{L}, L\right) d t\right) .
$$


Proof. At first we write $\zeta \stackrel{d}{=} \zeta_{1}+\zeta_{2}$ so that

$$
\begin{aligned}
V_{1}(x, L)= & \\
& \mathbb{E}_{(x, 1)}\left(\int_{0}^{\tau^{L} \wedge \zeta_{1}} e^{-\delta t} d L_{t}+\int_{\tau^{L} \wedge \zeta_{1}}^{\tau^{L} \wedge\left(\zeta_{1}+\zeta_{2}\right)} e^{-\delta t} d L_{t}+e^{-\delta\left(\zeta_{1}+\zeta_{2}\right)} R_{\zeta_{1}+\zeta_{2}}^{L} I_{\left\{\zeta_{1}+\zeta_{2}<\tau^{L}\right\}}\right) .
\end{aligned}
$$

Since the process $\left(R_{t}^{L}, \varepsilon_{t}\right)$ is a strong Markov process, we can write the second integral together with the terminal payment as $e^{-\delta \zeta_{1}} V_{2}\left(R_{\zeta_{1}}, \widehat{L}\right) I_{\left\{\zeta_{1}<\tau^{L}\right\}}$. The strategy $\widehat{L}$ is given by shifting the strategy $L$ by the starting time $\zeta_{1}$, i.e. $\widehat{L}_{t}=L_{\zeta_{1}+t}$. Taking the expectation with respect to $\zeta_{1}$ then gives the result.

The value functions of this state-dependent dividend maximization problem are defined as

$$
\begin{aligned}
V_{1}(x) & =\sup _{L \in \mathcal{L}} V_{1}(x, L), \\
V_{2}(x) & =\sup _{L \in \mathcal{L}} V_{2}(x, L) .
\end{aligned}
$$

As mentioned above, $V_{2}(x)$ is determined by Theorem 2.5. Combining Lemma 3.1 with the proof of a related dynamic programming principle (see Azcue \& Muler [7]) and replacing the arbitrary stopping time by $\zeta_{1}$, one gets that

$$
V_{1}(x)=\sup _{L \in \mathcal{L}} \mathbb{E}_{(x, 1)}\left(\int_{0}^{\tau^{L}} e^{-(\delta+\gamma) t} d L_{t}+\int_{0}^{\tau^{L}} e^{-(\delta+\gamma) t} \gamma V_{2}\left(R_{t}^{L}\right) d t\right),
$$

where $L \in \mathcal{L}$ can be taken as an admissible strategy (which in state 1 does not need to be a Markov control). The associated HJB equation for state 1 is then given by

$$
\begin{aligned}
& \max \left\{c f^{\prime}(x)+\int_{0}^{x} f(x-y) \alpha e^{-\alpha y} d y-(\delta+\gamma+\lambda) f(x)+\gamma V_{2}(x),\right. \\
&\left.1-f^{\prime}(x)\right\}=0 .
\end{aligned}
$$

The remainder of this section is devoted to the construction of an increasing and concave solution to (7).

It will turn out that it is without loss of generality to fix the strategies under consideration to be of state-dependent barrier type. I.e., if $\varepsilon_{t}=1$ we apply a barrier strategy at height $b_{1}$, and if $\varepsilon_{t}=2$ we apply a barrier strategy at height $b_{2}$. From the formulation of the switching problem, if $\varepsilon_{t}=2$ it will be preferable to distribute dividends in a more risky manner than in state 1 since killing is closer and because of discounting (i.e. in general $b_{1}>b_{2}$ ). This also matches the observation in Grandits [13] that $\varepsilon$-optimal barriers are decreasing in time.

We have to distinguish the following cases.

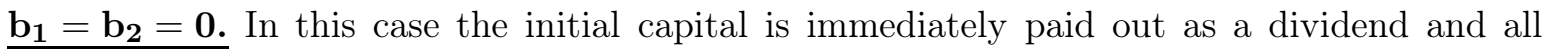
the incoming premium is also immediately paid out as dividends up to time $\min \left\{T_{1}, \zeta\right\}$, where $T_{1} \sim \operatorname{Exp}(\lambda)$ denotes the time of the first claim occurrence. Let $F_{\zeta}(s)$ denote the distribution function of $\zeta$, then the associated value is given by:

$$
\begin{aligned}
f_{(0,0)}(x) & =x+c \int_{0}^{\infty} \int_{0}^{t} e^{-\delta s}\left(1-F_{\zeta}(s)\right) d s \lambda e^{-\lambda t} d t \\
& =x+\frac{c(2 \gamma+\delta+\lambda)}{(\gamma+\delta+\lambda)^{2}} .
\end{aligned}
$$


At first we observe that this function can only be a candidate for the value function if $b_{2}=0$ is optimal for the second state.

Lemma 3.2. $f_{(0,0)}$ is a solution to (7) if $V_{2}(x)=x+\frac{c}{\delta+\gamma+\lambda}$ and if $(\delta+\gamma+\lambda)^{2}-\alpha c(2 \gamma+\delta+\lambda) \geq$ 0 or if $(\delta+\gamma+\lambda)^{2}-\alpha c(2 \gamma+\delta+\lambda)<0$ and $\alpha \lambda c(2 \gamma+\delta+\lambda)-(\delta+\lambda)(\delta+\gamma+\lambda)^{2} \leq 0$.

Proof. Since $f_{(0,0)}^{\prime}(x)=1$, we need to verify that the integro-differential part is non-positive. Plugging $f_{(0,0)}(x)$ into it, this part can be written as

$$
\frac{\lambda\left((\delta+\gamma+\lambda)^{2}-\alpha c(2 \gamma+\delta+\lambda)\right)\left(e^{-\alpha x}-1\right)-\alpha \delta x(\delta+\gamma+\lambda)^{2}}{\alpha(\delta+\gamma+\lambda)^{2}}=: J(x), \quad x \geq 0 .
$$

Obviously $J(x) \leq 0$ if $(\delta+\gamma+\lambda)^{2}-\alpha c(2 \gamma+\delta+\lambda) \geq 0$. Now suppose $(\delta+\gamma+\lambda)^{2}-\alpha c(2 \gamma+\delta+\lambda)<$ 0 , then $J(x)$ is concave. Therefore $J(0)=0$ is an upper bound for $J(x)$ if $J^{\prime}(0) \leq 0$. This is true if and only if

$$
\alpha \lambda c(2 \gamma+\delta+\lambda)-(\delta+\lambda)(\delta+\gamma+\lambda)^{2} \leq 0
$$

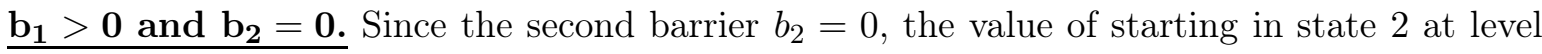

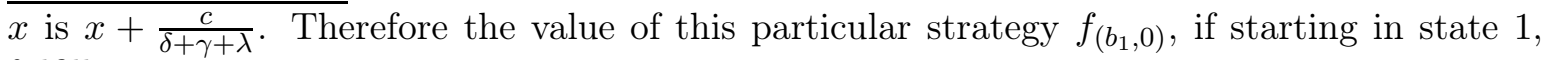
fulfills:

$$
\left\{\begin{array}{lr}
c f_{\left(b_{1}, 0\right)}^{\prime}(x)+\lambda \int_{0}^{x} f_{\left(b_{1}, 0\right)}(x-y) \alpha e^{-\alpha y} d y-(\delta+\gamma+\lambda) f_{\left(b_{1}, 0\right)}(x)+\gamma\left(x+\frac{c}{\delta+\gamma+\lambda}\right)=0, & 0 \leq x \leq b_{1}, \\
x-b_{1}+f_{\left(b_{1}, 0\right)}\left(b_{1}\right), & x>b_{1} .
\end{array}\right.
$$

Similar to the calculations in Section 2, we get that for $0 \leq x \leq b_{1}$ :

$$
f_{\left(b_{1}, 0\right)}(x)=D_{1}\left(b_{1}\right) e^{R_{1} x}+D_{2}\left(b_{1}\right) e^{R_{2} x}+\frac{\gamma x}{\delta+\gamma}+D_{3},
$$

where $R_{1}<0, R_{2}>0$ are the exponents determined in Section 2. Using $B:=\alpha c(2(\delta+\gamma)+$ $\lambda)-(\delta+\gamma+\lambda)^{2}$, the coefficients are

$$
\begin{aligned}
D_{1}\left(b_{1}\right) & =\frac{\left(R_{1}+\alpha\right)\left(\alpha^{2} \delta(\delta+\gamma)(\delta+\gamma+\lambda)+R_{2}\left(R_{2}+\alpha\right) \gamma B e^{R_{2} b_{1}}\right)}{\left.\alpha^{2}(\delta+\gamma)^{2}\right)(\delta+\gamma+\lambda)\left(R_{1}\left(R_{1}+\alpha\right) e^{R_{1} b_{1}}-R_{2}\left(R_{2}+\alpha\right) e^{R_{2} b_{1}}\right.}, \\
D_{2}\left(b_{1}\right) & =-\frac{\left(R_{2}+\alpha\right)\left(\alpha^{2} \delta(\delta+\gamma)(\delta+\gamma+\lambda)+R_{1}\left(R_{1}+\alpha\right) \gamma B e^{R_{1} b_{1}}\right)}{\left.\alpha^{2}(\delta+\gamma)^{2}\right)(\delta+\gamma+\lambda)\left(R_{1}\left(R_{1}+\alpha\right) e^{R_{1} b_{1}}-R_{2}\left(R_{2}+\alpha\right) e^{R_{2} b_{1}}\right.} \\
D_{3} & =\frac{\gamma(-\lambda(\delta+\gamma+\lambda)+\alpha c(2(\delta+\gamma)+\lambda)}{\left.\alpha(\delta+\gamma)^{2}\right)(\delta+\gamma+\lambda)} .
\end{aligned}
$$

This particular choice of coefficients gives that $f_{\left(b_{1}, 0\right)}$ is differentiable on $\mathbb{R}^{+}$.

Lemma 3.3. Assume that $V_{2}(x)=x+\frac{c}{\delta+\gamma+\lambda}$ and that $f_{(0,0)}$ does not solve (7). Let $H(b):=$ $f_{(b, 0)}^{\prime \prime}(b)$, then there exists a unique $b_{1}^{*}>0$ such that $H\left(b_{1}^{*}\right)=0$.

Proof. Similarly to the proof of Lemma 2.2, we get that

$$
H(0)=\frac{(\delta+\lambda)(\delta+\gamma+\lambda)^{2}-\alpha \lambda c(2 \gamma+\delta+\lambda)}{c(\delta+\gamma+\lambda)^{2}}
$$

and $\lim _{b \rightarrow \infty} H(b)=\frac{\delta R_{2}}{\delta+\gamma}>0$. We observe that $H(0)<0$ in this case and that $H(b)$ is continuous for $b \geq 0$. Further, one can see that using $\delta+\gamma+\lambda-\alpha c<0$ (which holds since a barrier in zero is optimal for state 2) one gets $H^{\prime}(b)>0$ for $b \geq 0$, which completes the proof. 
In the same way as in Lemma 2.3 one can derive that $f_{\left(b_{1}^{*}, 0\right)}$ is an increasing and concave function for $x \geq 0$. But nevertheless it is hard to analytically establish that

$$
c+\int_{0}^{x} f_{\left(b_{1}^{*}, 0\right)}(x-y) \alpha e^{-\alpha y} d y-(\delta+\gamma+\lambda) f_{\left(b_{1}^{*}, 0\right)}(x)
$$

$$
+\gamma\left(x+\frac{c}{\delta+\gamma+\lambda}\right) \leq 0, \quad x>b_{1}^{*}
$$

which would prove that $f_{\left(b_{1}^{*}, 0\right)}$ fulfills $(7)$. However there is numerical evidence that this is fulfilled in general.

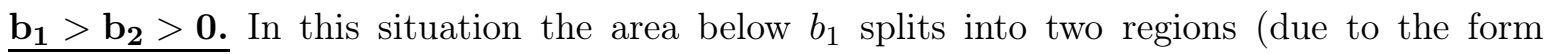
of $\left.V_{2}\right)$. The value $f_{\left(b_{1}, b_{2}\right)}(x)$ of this strategy is a solution to

$$
\left\{\begin{array}{cc}
c f_{\left(b_{1}, b_{2}\right)}^{\prime}(x)+\lambda \int_{0}^{x} f_{\left(b_{1}, b_{2}\right)}(x-y) \alpha e^{-\alpha y} d y-(\delta+\gamma+\lambda) f_{\left(b_{1}, b_{2}\right)}(x)+\gamma f_{b_{2}}(x)=0, & 0 \leq x \leq b_{2}, \\
c f_{\left(b_{1}, b_{2}\right)}^{\prime}(x)+\lambda \int_{0}^{x} f_{\left(b_{1}, b_{2}\right)}(x-y) \alpha e^{-\alpha y} d y-(\delta+\gamma+\lambda) f_{\left(b_{1}, b_{2}\right)}(x)+\gamma\left(x-b_{2}+f_{b_{2}}\left(b_{2}\right)\right)=0, \\
b_{2}<x \leq b_{1}, \\
x-b_{1}+f_{\left(b_{1}, b_{2}\right)}\left(b_{1}\right), \quad x>b_{1} .
\end{array}\right.
$$

One needs to be careful when dealing with the second integro-differential equation because the integration area includes the region below $b_{2}$. Nevertheless there are enough conditions (from smooth-pasting and the integrals) which can be translated into a linear system of equations, such that one can determine a differentiable solution of the following form:

$$
\begin{aligned}
& f_{\left(b_{1}, b_{2}\right)}(x)=\left(B_{1}\left(b_{1}, b_{2}\right)+x B_{2}\left(b_{2}\right)\right) e^{R_{1} x}+\left(B_{3}\left(b_{1}, b_{2}\right)+x B_{4}\left(b_{2}\right)\right) e^{R_{2} x} \\
&+B_{5} x+B_{6}, \quad 0 \leq x \leq b_{2}, \\
& f_{\left(b_{1}, b_{2}\right)}(x)=C_{1}\left(b_{1}, b_{2}\right) e^{R_{1} x}+C_{2}\left(b_{1}, b_{2}\right) e^{R_{2} x}+C_{3} x+C_{4}, \quad b_{2}<x \leq b_{1} .
\end{aligned}
$$

Although available in closed form, we can not display the voluminous explicit expressions for the involved coefficients here (they also include the coefficients of $f_{b_{2}}(x)$ ).

One can see that the right- and left- hand second derivatives of $f_{\left(b_{1}, b_{2}\right)}(x)$ at $x=b_{2}$ coincide since $f_{b_{2}}^{\prime}\left(b_{2}\right)=1$. Therefore the function $f_{\left(b_{1}, b_{2}\right)}(x)$ is twice differentiable on the whole interval $\left(0, b_{1}\right)$.

$\mathbf{b}_{\mathbf{1}}=\mathbf{b}_{\mathbf{2}}>\mathbf{0}$. For completeness we look at this case explicitly, which can be solved like the previous one, but the integro-differential part for $b_{2}<x \leq b_{1}$ disappears now and correspondingly calculations simplify. Setting $b=b_{1}=b_{2}>0$, the value of the associated strategy for $0 \leq x \leq b$ is given by

$$
f_{(b, b)}(x)=\left(E_{1}(b)+x E_{2}(b)\right) e^{R_{1} x}+\left(E_{3}(b)+x E_{4}(b)\right) e^{R_{2} x}+x E_{5}+E_{6} .
$$

Again the coefficients are determined by solving a linear system of equations such that $f_{(b, b)}^{\prime}(b)=$ 1.

Remark 3.1. Obviously a simple $\left(b_{1}, b_{2}\right)$-type strategy can only be a candidate for an optimal dividend strategy, if $b_{2}$ is optimal for the second state. Now suppose that $b_{2}^{*}>0$ (characterized via Theorem 2.2) is optimal, then a good candidate for $b_{1}$ would be the solution to $f_{\left(b_{1}, b_{2}^{*}\right)}^{\prime \prime}\left(b_{1}\right)=$ 0 . Like in the proof of Theorem 2.5, this condition together with $f_{\left(b_{1}, b_{2}^{*}\right)}^{\prime \prime \prime}\left(b_{1}\right)>0$ would imply 
that at $b_{1}$ the derivative attains a minimum with value 1 . This relates to the construction procedure for optimal band strategies in the infinite horizon case as described in Schmidli [17] , where one searches for minima equal to 1 of derivatives of solutions to the IDE part of the associated HJB equation.

Because of the elaborate structure of the coefficients of $f_{\left(b_{1}, b_{2}\right)}$ it seems out of reach to prove that a solution to $f_{\left(b_{1}, b_{2}\right)}^{\prime \prime}\left(b_{1}\right)=0$ exists in general and that the associated function solves (7) (in particular to verify that for $x>b_{1}^{*}$ the IDE part of the HJB equation evaluates to a non-positive value is intricate). We therefore propose the following procedure for deriving a candidate for $V_{1}$. For state 2 we already know the solution which is given in Theorem 2.5.

- If for state 2 the optimal barrier level $b_{2}^{*}=0$, from the conditions above either check whether $b_{1}^{*}=0$, or calculate $b_{1}^{*}>0$ using Lemma 3.3 and check whether

$$
\begin{aligned}
c+\int_{0}^{x} f_{\left(b_{1}^{*}, 0\right)}(x-y) \alpha e^{-\alpha y} d y-(\delta+\gamma+\lambda) f_{\left(b_{1}^{*}, 0\right)}(x) & \\
& +\gamma\left(x+\frac{c}{\delta+\gamma+\lambda}\right) \leq 0, \quad x>b_{1}^{*} .
\end{aligned}
$$

If this is true, $f_{\left(b_{1}^{*}, 0\right)}$ is an increasing and concave solution to (7).

- If $b_{2}^{*}>0$, use the case $b_{1} \geq b_{2}>0$. Determine $b_{1}^{*}>b_{2}^{*}$ as a solution to $f_{\left(b_{1}, b_{2}\right)}^{\prime \prime}\left(b_{1}\right)=0$ and check whether it is a concave solution to (7).

If a simple state-dependent barrier strategy solves the dividend problem, the above procedure will deliver the solution. Under this type of strategy the controlled reserve process is a strong Markov process with respect to the filtration generated by the risk reserve process and the external state process $\varepsilon_{t}$.

We conclude this section with the following verification theorem.

Theorem 3.4. Let $h: \mathbb{R} \rightarrow \mathbb{R}^{+}$with $h(x)=0$ for $x<0$, be an increasing, differentiable and concave solution to (7) which corresponds to an admissible strategy $L^{*}$ for state 1 and the optimal strategy for state 2. Then $h(x)=V_{1}(x)$ and $L^{*}$ is optimal if starting in state 1 .

Proof. Let $L$ be an admissible strategy. We can follow the proof of Theorem 2.4 to arrive at

$$
\mathbb{E}_{(x, 1)}\left(\int_{0}^{\tau^{L}} e^{-(\delta+\gamma) s} d L_{s}+\int_{0}^{\tau^{L}} e^{-(\delta+\gamma) s} \gamma V_{2}\left(R_{s-}^{L}\right) d s\right) \leq h(x) .
$$

Since $V_{2}(x) \geq V_{2}(x, L)$ we get, by incorporating the time split $\zeta \sim \zeta_{1}+\zeta_{2}$,

$$
V_{1}(x, L)=\mathbb{E}_{(x, 1)}\left(\int_{0}^{\tau^{L} \wedge \zeta} e^{-\delta s} d L_{s}+e^{-\delta \zeta} R_{\zeta}^{L} I_{\left\{\zeta<\tau^{L}\right\}}\right) \leq h(x) .
$$

Now using the strategy $L^{*}$ while being in state 1 and the optimal strategy for state 2 we have an equality in (8) which completes the proof. 


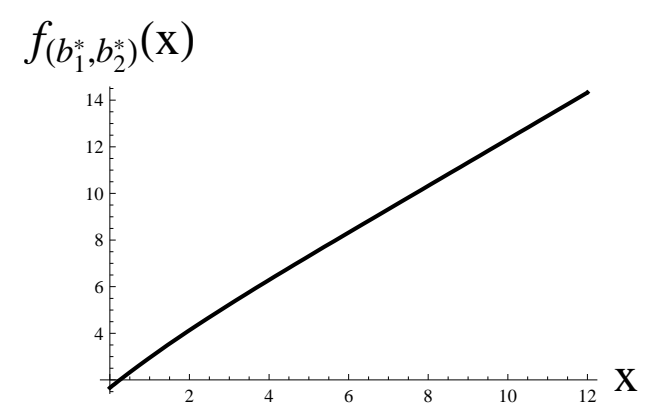

Figure 7: $f_{\left(b_{1}^{*}, b_{2}^{*}\right)}$ for $x \in[0,10)$

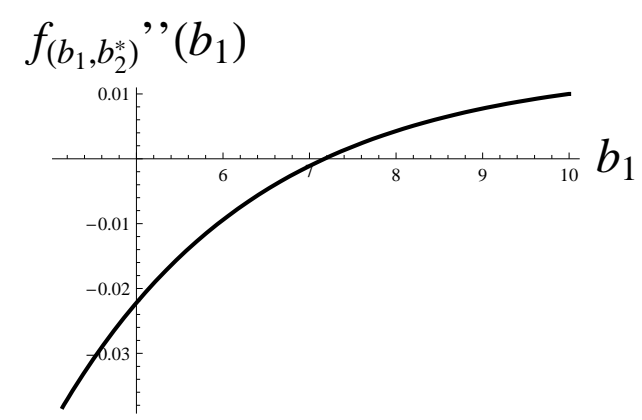

Figure 9: $f_{\left(b_{1}, b_{2}^{*}\right)}^{\prime \prime}\left(b_{1}\right)$ for $b_{1} \in\left(b_{2}^{*}, 10\right)$

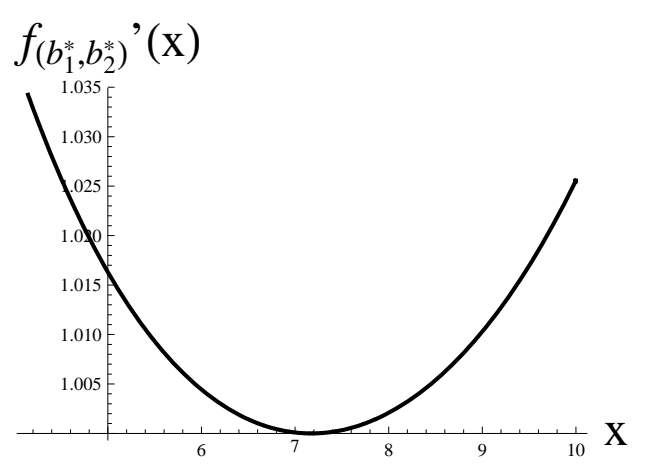

Figure 8: $f_{\left(b_{1}^{*}, b_{2}^{*}\right)}^{\prime}(x)$, for $x \in\left(b_{2}^{*}, 10\right)$

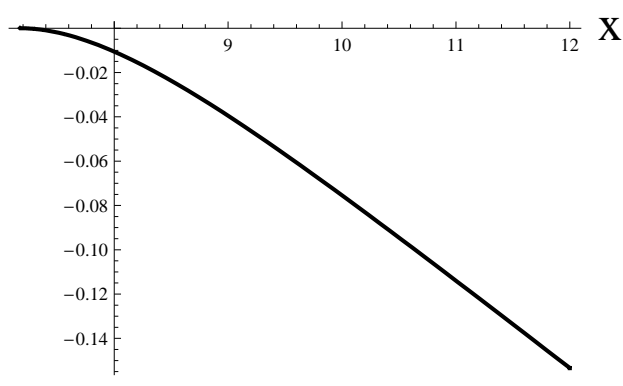

Figure 10: IDE part of HJB eq., $x \in$ $\left(b_{1}^{*}, 12\right)$

\subsection{Illustrations}

Example 1. For the first example we choose a parameter set which delivers positive barriers for both states: $c=6.5, \lambda=5, \alpha=1, \gamma=1, \delta=0.04$. For state 2 we numerically calculate $b_{2}^{*}=4.13685$ and have that $V_{2}(x)=f_{b_{2}^{*}}(x)$. One then determines $b_{1}^{*}=7.17286$ as a solution to $f_{\left(b_{1}, b_{2}^{*}\right)}^{\prime \prime}\left(b_{1}\right)=0$.

Figure 7 shows $f_{\left(b_{1}^{*}, b_{2}^{*}\right)}(x)$. Figure 10 displays the IDE part of the HJB equation evaluated at $f_{\left(b_{1}^{*}, b_{2}^{*}\right)}$ for $x>b_{1}^{*}$. Since it is negative the HJB equation is fulfilled and one can deduce from Theorem 3.4 that it is the value function of the dividend maximization problem, if starting in state 1. Furthermore Figure 8 illustrates that the minimum of the derivative is exactly equal to 1 at $b_{1}^{*}$. Figure 9 contains the graph of $f_{\left(b_{1}, b_{2}^{*}\right)}^{\prime \prime}\left(b_{1}\right)$ as a function of the barrier for state 1 . The optimal state-dependent strategy, namely to use the barrier strategy $b_{1}^{*}$ up to the random time $\zeta_{1}$, and from then on $b_{2}^{*}$, is depicted in Figure 11.

Example 2. As a second example we reduce the premium intensity to $c=5.5$ and leave all other parameters unchanged. For state 2 the optimal barrier is now zero, i.e. $V_{2}(x)=x+\frac{c}{\delta+\gamma+\lambda}$ (one can observe the same effect by changing $\gamma=1$ to $\gamma=2$ (earlier killing) while keeping $c=6.5)$. One then calculates $b_{1}^{*}=3.70785$ as a solution to $f_{\left(b_{1}, 0\right)}^{\prime \prime}\left(b_{1}\right)=0$.

Here the crucial plot is Figure 15. As the IDE part is negative to the right of the barrier, we have that $f_{\left(b_{1}^{*}, 0\right)}$ is the value function, a plot of which is given in Figure 12. Figures 13 and 14 show $f_{\left(b_{1}^{*}, 0\right)}^{\prime}(x)$ and $f_{\left(b_{1}, 0\right)}^{\prime \prime}\left(b_{1}\right)$, illustrating that the minimum of the derivative is equal to 1 and the unique root of this specific second derivative.

Example 3. If we keep $c=5.5$ and $\lambda=5$, but choose $\gamma=3$, we get $V_{2}=x+\frac{c}{\delta+\gamma+\lambda}$ and a barrier in zero is then also optimal for state 1, i.e. $V_{1}(x)=x+\frac{c(2 \gamma+\delta+\lambda)}{(\gamma+\delta+\lambda)^{2}}$. 


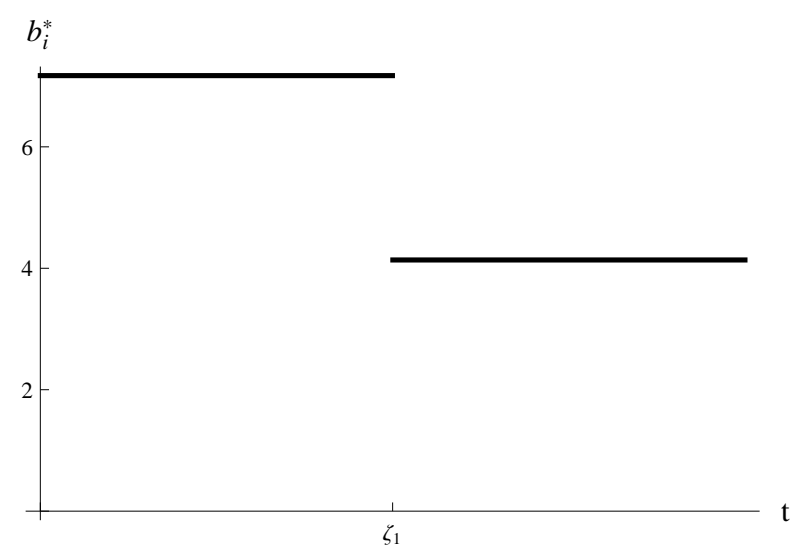

Figure 11: $b_{i}^{*}$ for $i=1,2$

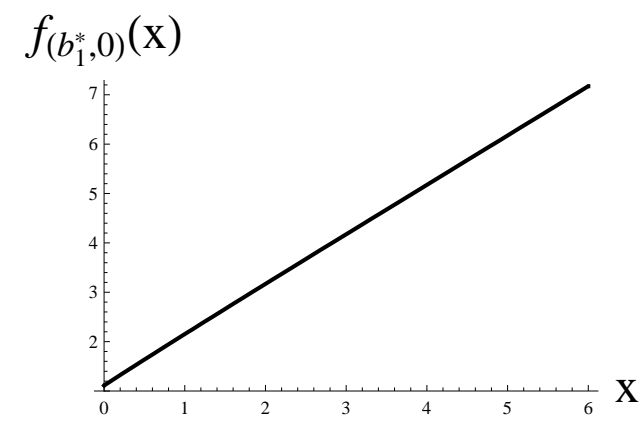

Figure 12: $f_{\left(b_{1}^{*}, 0\right)}$ for $x \in[0,6)$

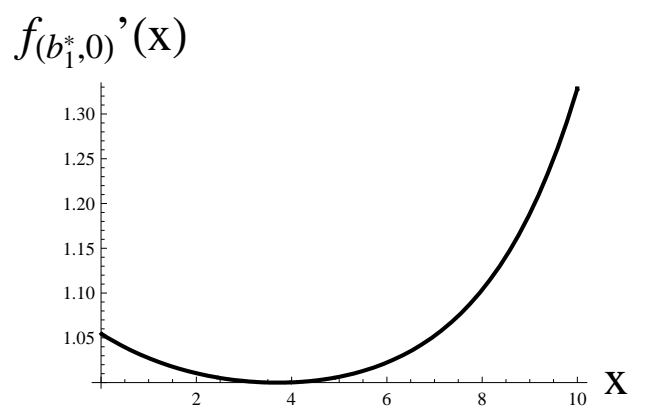

Figure 13: $f_{\left(b_{1}^{*}, 0\right)}^{\prime}(x)$, for $x \in(0,10)$

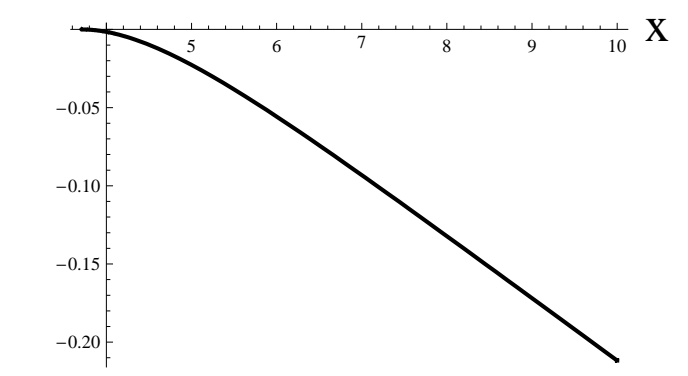

Figure 15: IDE-HJB for $x \in\left(b_{1}^{*}, 10\right)$
Figure 14: $f_{\left(b_{1}, 0\right)}^{\prime \prime}\left(b_{1}\right)$ for $b_{1} \in(0,10)$

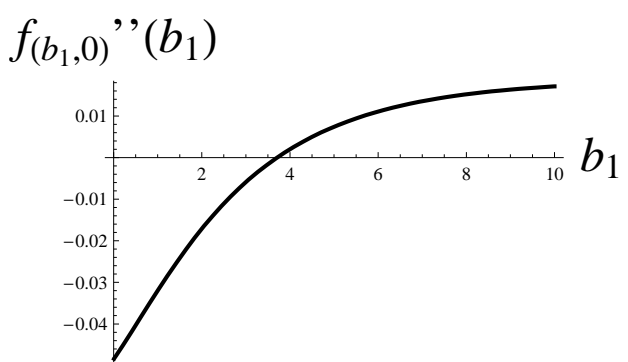




\section{Conclusion and Outlook}

In this contribution we identified and discussed a control problem in insurance, namely we extended the classical optimal dividend problem in insurance by the non-standard feature of having a random time horizon. We explicitly solved this problem for the case when both the time horizon and the individual claim sizes are exponentially distributed. We then extended the solution procedure to the case of an $\operatorname{Erlang}(2)$ time horizon by introducing an external state process and under the assumption that the state of this external process is observable, in which case the problem gets the flavor of a Markov regime switching problem. A natural next step for future research will be to apply some filtering technique to remove the (somewhat artificial) assumption of observability of the state process to explicitly solve the Erlang(2) time horizon problem (in which case there is an interpretation in terms of a Hidden Markov Model). The optimal strategy may then be expected to be of a time-dependent barrier form, where the value of the barrier at each time point arises from mixing over the optimal barrier values of the two states and the mixing probabilities are given by the probability to be in the respective state at that time point. This procedure should then naturally extend to the Erlang(n) case, and the latter can then also be used to approximate the deterministic finite time horizon dividend problem. Since the diffusion approximation for the risk reserve process is a limit of the classical insurance risk process, this program may also give additional tools for analyzing the behavior of the optimal time-dependent barrier for the diffusion model studied by Grandits [13].

However, in view of the complexity of the involved expressions, already the Erlang(2) case is intricate and correspondingly the explicit identification of the optimal solution candidate for general Erlang(n) horizons and the formal verification of its optimality are expected to be a challenging problem.

\section{References}

[1] H. Albrecher, N. Bäuerle and S. Thonhauser. Optimal dividend pay-out in random discrete time. Statistics and Risk Modeling. 28 (3), 251-276, (2011).

[2] H. Albrecher and J. Hartinger. On the non-optimality of horizontal barrier strategies in the Sparre Andersen model. Hermis Journal on Computer Mathematics and Applications. 7, 109-122, 2006.

[3] H. Albrecher and S. Thonhauser. Optimality results for dividend problems in insurance. Rev. R. Acad. Cienc. Exactas Fís. Nat. Ser. A Mat. RACSAM. 103 (2), 295-320, (2009).

[4] S. Asmussen and H. Albrecher. Ruin probabilities. (World Scientific, New Jersey, 2010).

[5] S. Asmussen, F. Avram, and M. Usabel. Erlangian approximations for finite-horizon ruin probabilities. ASTIN Bulletin. 32 (2), 267-281, (2002).

[6] B. Avanzi. Strategies for dividend distribution: A review. N. Am. Actuar. J.. 13 (2), 217-251, (2009).

[7] P. Azcue and N. Muler. Optimal reinsurance and dividend distribution policies in the Cramér-Lundberg model. Math. Finance. 15 (2), 261-308, (2005).

[8] P. Carr. Randomization and the American put. Rev. Financ. Stud.. 11 (3), 597-626, (1998). 
[9] M. H. A. Davis. Markov models and optimization. (Chapman \& Hall, London, 1993).

[10] B. De Finetti. Su un' impostazione alternativa dell teoria collettiva del risichio. Transactions of the XVth congress of actuaries. (II), 433-443, (1957).

[11] W. H. Fleming and H. M. Soner. Controlled Markov processes and viscosity solutions. (Springer, New York, 1993).

[12] J. Grandell. Aspects of risk theory. (Springer, New York, 1991).

[13] P. Grandits. Optimal consumption in a Brownian model with absorption and finite time horizon. Preprint, 31 pages, (2009).

[14] N. Kulenko and H. Schmidli. Optimal dividend strategies in a Cramér-Lundberg model with capital injections. Insurance Math. Econom.. 43 (2), 270-278, (2008).

[15] R. Loeffen and J-F. Renaud. De Finetti's optimal dividends problem with an affine penalty function at ruin. Insurance Math. Econom.. 46 (1), 98-108, (2010).

[16] T. Rolski, H. Schmidli, V. Schmidt, and J. Teugels. Stochastic processes for insurance and finance. (Wiley, 1999).

[17] H. Schmidli. Stochastic Control in Insurance. (Springer, New York, 2008).

[18] L. R. Sotomayor and A. Cadenillas. Classical and singular stochastic control for the optimal dividend policy when there is regime switching. Insurance Math. Econom.. 48 (3), 344-354, (2011). 